JOURNAL of

MAINE MEDICAL CENTER Journal of Maine Medical Center

Volume 2

Issue 1 Volume 2, Issue 1 (January 2020)

Article 8

2020

\title{
Fresh Fruits and Vegetables Really Do Keep the Doctor Away: Symptomatic Vitamin Deficiency in a Middle Aged Man
}

\author{
Matthew J. Turnquist MD \\ Maine Medical Center
}

Et al.

Follow this and additional works at: https://knowledgeconnection.mainehealth.org/jmmc

Part of the Dietetics and Clinical Nutrition Commons, Preventive Medicine Commons, and the Primary Care Commons

\section{Recommended Citation}

Turnquist, Matthew J. MD and Holt, Christina MD, MSc (2020) "Fresh Fruits and Vegetables Really Do Keep the Doctor Away: Symptomatic Vitamin Deficiency in a Middle Aged Man," Journal of Maine Medical Center. Vol. 2 : Iss. 1 , Article 8.

Available at: https://knowledgeconnection.mainehealth.org/jmmc/vol2/iss1/8 https://doi.org/10.46804/ 2641-2225.1018

The views and thoughts expressed in this manuscript belong solely to the author[s] and do not reflect the opinions of the Journal of Maine Medical Center or MaineHealth.

This Case Report is brought to you for free and open access by Maine Medical Center Department of Medical Education. It has been accepted for inclusion in the Journal of Maine Medical Center by an authorized editor of the MaineHealth Knowledge Connection. For more information, please contact Dina McKelvy mckeld1@mmc.org.

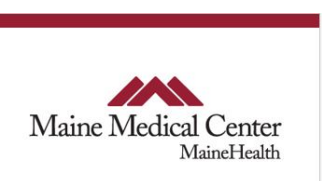


Fresh Fruits and Vegetables Really Do Keep the Doctor Away: Symptomatic Vitamin Deficiency in a Middle Aged Man

\section{Authors}

Matthew J. Turnquist MD and Christina Holt MD, MSc 


\title{
Fresh Fruits and Vegetables Really Keep the Doctor Away: Symptomatic Vitamin Deficiency in a Middle-Aged Man, A Case Study
}

\author{
Matthew Turnquist, MD, Christina Holt, MD, MSc \\ Department of Family Medicine, Maine Medical Center, Portland, ME
}

\begin{abstract}
Introduction: We present a clinical case of symptomatic vitamin deficiencies, typically seen only in resourcelimited environments or in alcoholics. An adult male presented with frequent oral intake but limited diet of prepared foods, as well as co-occurring pernicious anemia.
\end{abstract}

Clinical findings: The patient presented with complaints of ascending paresthesia, near syncope associated with standing, lower extremity edema, and hyperpigmentation on his anterior legs bilaterally, which had been present for years.

\begin{abstract}
Diagnoses, Interventions, and Outcomes:

The patient was found to have mixed deficiencies in vitamin B12, thiamine, and folic acid. These results were attributed to a combination of nutritional deficiency due to a diet of exclusively cooked foods and pernicious anemia. The patient was treated with parenteral and oral vitamin supplementation, and he was noted to improve. He was lost to follow up.
\end{abstract}

Conclusions: $\quad$ This case highlights the importance of social and nutritional history in health maintenance, as well as the clinical significance of prolonged mixed vitamin deficiencies.

Keywords: $\quad$ mixed vitamin deficiency, pernicious anemia, hyperpigmentation, medical history

\section{CASE PRESENTATION}

A 39-year-old male presented with complaints of paresthesia, edema, and near syncope. He reported no medical care for 4 years, but a medical history of gout and hypertension. He was taking no medications or supplements.

The patient reported one month of lower extremity edema and bilateral paresthesia that occurred when standing or walking, which was relieved with sitting and elevation of his legs. His symptoms progressively increased since onset. He reported recent near-syncope and nausea with standing, including several recent episodes of emesis.

Correspondence: Matthew Turnquist, MD

Maine Medical Center, Department of Family Medicine

5 Bucknam Rd, Falmouth, ME 04105

mturnquist@mmc.org
Incidentally, he reported patchy discoloration of his legs. This discoloration was previously noted by his physicians, who did not give the patient an explanation for the symptom.

He lived alone and reported that his diet consisted mostly of 2-3 daily meals of "takeout and fast food." He reported drinking four alcoholic drinks nightly and smoking marijuana 2-3 times weekly. He denied using any other non-prescription drugs. He was not aware of any toxic or work-related chemical exposures that may have contributed to his symptoms.

The patient was pale, obese, and tachycardic (heart rate ranged from 98 to 114 beats per minute), but his vital signs were otherwise normal. He exhibited no jugular vein distention. He had severe pitting edema of his lower extremities bilaterally to the knees. He 
had no sensory loss in his lower extremities when seated with legs elevated, but he had numbness in his lower extremities when standing or walking. $\mathrm{He}$ was noted to have tan to brown patches of hyperpigmentation on his anterior legs bilaterally. No tremor or weakness was noted. The remainder of his physical exam was unremarkable.

Initial workup in the emergency department (ED) focused on several possible causes of edema, peripheral neuropathy, and pre-syncope. A complete workup was done for hemolytic anemia or acute bleeding. A diagnosis of symptomatic vitamin deficiency was not initially considered upon presentation to the ED. After reviewing his laboratory values (Table 1), his symptoms were attributed to a mixed vitamin deficiency with elements of pernicious anemia, "wet" beriberi (thiamine deficiency affecting the cardiovascular system and resulting in peripheral edema), and folic acid deficiency. This diagnosis was confirmed by his low levels of serum vitamins and positive testing for intrinsic factor antibody. Alternative causes for edema and neuropathy were ruled out by his borderline hemoglobin A1c, thyroidstimulating hormone (TSH), and renal function. $\mathrm{He}$ was treated with parenteral supplementation of vitamin B12 and thiamine, as well as oral folic acid. His neuropathy and edema were noted to begin resolving with treatment, and he was discharged home on supplementation. Unfortunately, he was lost to follow up after discharge to his primary care physician.

\section{DISCUSSION}

This case provides an example of a patient who suffered multiple vitamin deficiencies that were clinically significant in the context of excessive intake of processed foods, alcohol use disorder, and chronic underlying autoimmunity. The patient's previous providers overlooked his lower extremity hyperpigmentation as a clinical symptom of deficiency. His ED workup focused on hemolytic anemia or bleeding, rather than on nutritional or autoimmune deficiency. This case highlights the importance of taking the social and nutritional history of patients.

Pernicious anemia results from decreased absorption of vitamin B12. This decreased absorption is due to autoimmunity and progressive loss of parietal cells in the body and fundus of the stomach responsible for producing intrinsic https://knowledgeconnection.mainehealth.org/jmmc/vol2/iss $1 / 8$ factor antibody, a glycoprotein needed for vitamin B12 absorption in the small intestine. ${ }^{1}$ Vitamin B12 is needed for both erythropoiesis and myelin synthesis, and its deficiency results in anemia and neuropathy. The resulting neuropathy is "lengthdependent," first affecting the long axons of the lower extremities. ${ }^{2}$ While we typically consider vitamin B12 deficiency in cases of persistent distal polyneuropathy and memory deficits, the diagnosis should also be considered in cases of intermittent symptoms, particularly with increasing patterns of progression. Additionally, lower extremity hyperpigmentation has been associated with vitamin B12 deficiency ${ }^{3}$ and may have been an early clinical sign of this patient's disease. This patient's combination of length-dependent neuropathy, hyperpigmentation, low serum vitamin B12, and positive antibody testing confirms his diagnosis. Both vitamin B12 and folate deficiency correlate with elevated homocysteine, ${ }^{3,4}$ which may lead to the formation of deep vein thrombosis (DVT) due to hypercoagulability. ${ }^{5}$ While this patient did not have DVT, this diagnosis should be considered in patients presenting with a combination of either vitamin B12 or folate deficiency and lower-extremity edema.

Thiamine deficiency most commonly occurs with poor dietary intake in the context of severe alcohol abuse. It may also result from diets lacking whole grains or fortified foods. ${ }^{6}$ Processing of grains removes the vitamin-containing husk and may lead to vitamin deficiency in the absence of other dietary sources. Thiamine is a critical cofactor in cellular metabolism. Its deficiency can result in a range of symptoms related to oxidative stress in the neurological and cardiovascular systems, ${ }^{6}$ including Wernicke's encephalopathy, "dry" beriberi involving the peripheral nerves, and "wet" beriberi involving the cardiovascular system. In this case, low serum thiamine, tachycardia, and peripheral edema were consistent with "wet" beriberi. The patient improved with therapy, confirming the diagnosis. Thiamine deficiency may have also exacerbated his neuropathy.

Although folic acid is in a variety of foods, it is temperature sensitive and may be destroyed or leeched into cooking water when prepared. ${ }^{4}$ Diets depending entirely on cooked foods, regardless of variety, are a risk factor for folate deficiency. Folic acid is critical to DNA replication and cellular division. Folic acid deficiency may present with macrocytic anemia, as in this patient, or with more 
Table 1. Diagnostic Laboratory Testing Evaluating Patient with Paresthesia, Edema, and Pre-Syncope.

\begin{tabular}{|l|l|l|}
\hline Test & & \multicolumn{1}{l}{ Normal Range } \\
\hline $\begin{array}{l}\text { Complete blood count } \\
\text { Hemoglobin }\end{array}$ & $8.2 \mathrm{~g} / \mathrm{dL}$ & \\
MCV & $124.9 \mathrm{fL}$ & $13.0-17.4 \mathrm{~g} / \mathrm{dL}$ \\
RDW - SD & $62.1 \mathrm{fL}$ & $\begin{array}{l}82.0-100.0 \mathrm{fL} \\
37-48 \mathrm{fL}\end{array}$ \\
\hline Smear & $1+$ macrocytes & \\
& $1+$ polychromasia & \\
& $1+$ ovalocytes & \\
\hline Reticulocyte count & $1+$ teardrop cells & $23,000-100,000 / \mu \mathrm{L}$ \\
\hline Hemoglobin A1c & $12,000 / \mu \mathrm{L}$ & $4.5-5.7 \%$ \\
\hline Vitamin B12 & $6.0 \%$ & $232-1245 \mathrm{pg} / \mathrm{mL}$ \\
\hline Thiamine & $202 \mathrm{pg} / \mathrm{mL}$ & $38-122 \mathrm{mcg} / \mathrm{L}$ \\
\hline Folate & $34 \mathrm{mcg} / \mathrm{L}$ & $4.8-20.0 \mathrm{ng} / \mathrm{mL}$ \\
\hline Intrinsic factor antibody & $2.0 \mathrm{ng} / \mathrm{mL}$ & $\mathrm{Neg}$ \\
\hline Comprehensive metabolic panel & Positive & \\
\hline Total protein & & $5.9-8.4 \mathrm{~g} / \mathrm{dL}$ \\
Albumin & $6.0 \mathrm{~g} / \mathrm{dL}$ & $3.2-5.2 \mathrm{~g} / \mathrm{dL}$ \\
BUN & $3.1 \mathrm{~g} / \mathrm{dL}$ & $6-19 \mathrm{mg} / \mathrm{dL}$ \\
Creatinine & $11 \mathrm{mg} / \mathrm{dL}$ & $0.50-1.30 \mathrm{mg} / \mathrm{dL}$ \\
\hline TSH & $0.46 \mathrm{mg} / \mathrm{dL}$ & $0.27-4.20 \mathrm{mU} / \mathrm{L}$ \\
\hline
\end{tabular}

BUN, blood urea nitrogen; MCV, mean corpuscular volume; RDW -SD, red blood cell distribution width -standard deviation; TSH, thyroid-stimulating factor.

superficial signs, such as mucosal ulcerations. His low serum folate level and improvement with therapy confirmed the diagnosis.

Severe vitamin deficiencies are most often considered clinically in circumstances of extremely poor oral intake or alcohol abuse. They are also a risk for people with underlying autoimmune disease or whose diets lack raw foods, contain only processed grains, or have underlying autoimmune disease. By the time clinical symptoms arise, patients are generally quite deficient. Our case presented with symptoms of severe combined vitamin deficiency syndromes. A thorough nutritional and alcohol history might have identified the patient's deficiency before he presented in the ED or lead his physicians to more quickly definitively diagnose him when he arrived.

\section{Conflicts of Interest None}

\section{REFERENCES}

1. Ammuri W, Tazi ZM, Harmouche H, Maamar M, Adnaoui M. Venous thromboembolism and hyperhomocysteinemia as first manifestation of pernicious anemia: a case series. J Med Case Rep 2017;11(1):250. doi: 10.1186/s13256-017-1415-z.

2. Bizzaro N, Antico A. (2014). Diagnosis and classification of pernicious anemia. Autoimmun Rev 2014;13(4-5):565-568. doi: 10.1016/j.autrev.2014.01.042.

3. Callaghan BC, Price RS, Feldman EL. Distal symmetric polyneuropathy: a review. JAMA 2015;314(20)2172-2181.

4. DeFilippis EM, Petersen AM, Yialamas MA. Progressive weakness and memory impairment in a middle-aged man. JAMA 2018;320(2):197-198. doi: 10.1001/jama.2018.6474.

5. Liu D, Ke Z, Luo J. Thiamine deficiency and neurodegeneration: the interplay among oxidative stress, endoplasmic reticulum stress and autophagy. Mol Neurobiol 2017;54(7):5440-5448. doi: 10.1007/s12035-016-0079-9.

6. Schrier SM. Clinical manifestations and diagnosis of vitamin B12 and folate deficiency. UptoDate website. https://www.uptodate. $\mathrm{com} /$ contents/clinical-manifestations-and-diagnosis-of-vitaminb12-and-folate-deficiency. Updated August 27, 2018. Accessed October 19, 2018.

7. Snow CF. Laboratory diagnosis of vitamin B12 and folate deficiency: a guide for the primary care physician. Arch Intern Med 1999;159(12):1289-1298. 